\title{
EL INGRESO FAMILIAR COMO DETERMINANTE DE LA ASISTENCIA ESCOLAR DE LOS JÓVENES EN MÉXICO
}

\author{
Gloria Mancha ${ }^{\mathrm{a}}$ y Edgardo Ayala ${ }^{\mathrm{b}}$
}

Fecha de recepción: 9 de junio de 2019. Fecha de aceptación: 6 de enero de 2020 .

$$
\text { http://doi.org/10.22201/iiec.20078951e.2020.201.69395 }
$$

Resumen. El presente trabajo estima el impacto del ingreso familiar en la asistencia escolar de los jóvenes en México en un contexto longitudinal. Con base en los paneles rotativos de la Encuesta Nacional de Ocupación y Empleo (ENOE), se estima un modelo econométrico probit con efectos fijos para determinar la probabilidad de que un joven de 14 a 18 ańos que vive con sus padres y asiste a la escuela -continúe el siguiente año-, controlando por las características del joven, de su familia, del entorno y cambios temporales. Se encontró evidencia de que la probabilidad de que un joven continúe estudiando aumenta con el ingreso familiar, y este efecto positivo es relativamente más importante en los primeros deciles de ingreso.

Palabras clave: ingreso familiar; jóvenes; asistencia escolar; educación media; educación media superior.

Clasificación JEL: E24; C23; C33; I21; I22; J13.

\section{FAMILY INCOME AS A DETERMINANT OF YOUNG PEOPLE'S SCHOOL ATTENDANCE IN MEXICO}

\begin{abstract}
This paper calculates the impact of family income on young people's school attendance in Mexico, in a longitudinal context. Based on the rotating panels of the National Occupation and Employment Survey (ENOE), a probit econometric model with fixed effects is used to determine the likelihood that a 14- to 18-year-old who lives with their parents and attends school will continue to attend in the following year; the model is controlled by the young person's characteristics, their family, the environment and various temporary changes. Evidence was found that the probability of a young person continuing to attend school increases with family income, and this positive effect is relatively more important in the first deciles of income.

Key Words: family income; young people; school attendance; secondary school; high school education.

\footnotetext{
${ }^{a}$ Universidad Autónoma de Nuevo León, México; ${ }^{\mathrm{b}}$ Escuela Nacional de Ciencias Sociales del Tecnológico de Monterrey, México. Correos electrónicos: glmancha@gmail.com y edgardo@itesm.mx, respectivamente.
} 


\section{INTRODUCCIÓN}

En las últimas décadas, los jóvenes se han convertido en un grupo vulnerable que enfrenta altas tasas de desempleo, informalidad y precariedad laboral y la falta de oportunidades de educación y de acceso al sistema de protección social, de salud, entre otros (Beck, 1998; CEPAL, 2008; Gruber, 2009; IMJ, 2007; Miranda y Salvia, 1998; Mancha, 2011). Sin embargo, esta situación no es homogénea. Los jóvenes enfrentan importantes diferencias intrageneracionales entre aquellos que tienen acceso a una educación de calidad, empleos bien remunerados y de alto valor agregado, a las tecnologías de la información y la comunicación (TICs), a la salud, etcétera, frente a quienes no lo tienen (Hopenhayn, 2008). Entre más amplias sean las diferencias, mayor será la polarización social y exclusión de individuos de una misma generación, y por tanto mayores serán los costos sociales y económicos para el país (oit, 2005).

Estudios como el realizado por De Gregorio y Lee (2002) encuentran que mientras mayor y más igualitaria sea la educación, más equitativa es la distribución del ingreso a lo largo de la vida; en palabras de Formichella (2011, p. 16) citando a Gasparini (2001) y Guadagni (2007) "la equidad educativa puede aumentar la equidad económica”. De acuerdo a Tilak (2002), varios estudios en todo el mundo señalan una correlación positiva entre mayor escolaridad y mayores ingresos; según Tilly (2003), quienes poseen más conocimiento tienen más opciones de desarrollo.

Así, la educación es vista como una de las formas para disminuir la desigualdad y la pobreza. Sin embargo, una mayor educación (medida en años de escolaridad), depende de una serie de factores tanto internos como externos al individuo, que conducen a que se forme una brecha entre quienes obtienen mayor educación y aquellos que no pueden (o no quieren) continuar su trayectoria educativa. En la teoría de la desigualdad, estos aspectos pueden dividirse en factores sobre los que se tiene control como responsabilidad individual o esfuerzo personal, y factores predeterminados sobre los que no se tiene control como raza, género, condiciones familiares, capital cultural, herencia, ingreso familiar y otros (Roemer, 1998).

El objetivo de este trabajo es estudiar el efecto sobre la asistencia escolar de un joven de 14 a 18 años dada una situación de la que no tiene control: el ingreso familiar que, como se señala, es un determinante de la desigualdad de oportunidades en educación. La mejor forma de realizar este tipo de análisis es usando información longitudinal, es decir, bases de datos que siguen 
la asistencia escolar del individuo y el ingreso familiar a lo largo del tiempo. Sin embargo, en muchos países, como es el caso de México, no existe registro de este tipo de información, por lo que las investigaciones emplean bases de datos de corte transversal. Si bien los datos de sección cruzada son más fáciles de recolectar y procesar tienen el inconveniente de presentar observaciones en el mismo periodo de tiempo para todas las variables, lo que puede presentar problemas de endogeneidad o doble causalidad entre el ingreso familiar y la asistencia escolar, obligando a plantear importantes supuestos en la distribución de los errores y en la pérdida de eficiencia en los estimadores.

Este estudio contribuye al análisis de la asistencia escolar mediante la estimación un modelo microeconométrico longitudinal aprovechando la estructura de panel rotativo de datos de la Encuesta Nacional de Ocupación y Empleo (ENOE) de México, utilizando el ingreso familiar del joven con un ańo de rezago como determinante de la asistencia escolar. Este panel rotativo de un año y el rezago en el ingreso permite aislar, al menos parcialmente, la endogeneidad del ingreso en la asistencia escolar de los jóvenes en México y obtener estimadores sin sesgo del efecto del ingreso familiar en la probabilidad de que los jóvenes asistan a la escuela.

Las estimaciones indican que el acceso y la permanencia escolar de los jóvenes (medido a través de que los jóvenes que están estudiando continúen asistiendo a la escuela el siguiente año) es determinado en parte por el ingreso familiar, lo que sugiere la existencia de una brecha intrageneracional en educación basada en este factor.

El artículo se organiza de la siguiente forma: en la segunda sección se presenta la asistencia educativa en México; en la tercera se desarrolla la revisión de literatura; en la cuarta se discuten la metodología, las variables y los datos. Finalmente, la quinta sección presenta los resultados y la sexta concluye.

\section{ASISTENCIA EDUCATIVA EN MÉXICO: ALGUNOS HECHOS ESTILIZADOS}

En México, los jóvenes de entre 14 y 18 años enfrentan condiciones desiguales, lo que afecta sus oportunidades de educación y contribuye a aumentar la brecha de escolaridad. Por ejemplo, en 2015 se contabilizaban 9585389 jóvenes de esta edad; de estos, 7669472 asistían a la escuela, es decir, 20\% de los jóvenes en este rango de edad no asistían a la escuela. 
Al desglosar por edad, es posible observar cómo aumenta el porcentaje de jóvenes que no asisten a la escuela a mayor edad. Como se deriva del cuadro 1 , los jóvenes de 14 años que asisten a la escuela representa el 93\% del total; el restante $7 \%$ no asiste. Por su parte, a los 18 años, sólo asiste a la escuela $64.68 \%$, y no lo hace $35.32 \%$.

Cuadro 1. México. Asistencia escolar por edad (14 a 18 años) en 2015.

Valores absolutos y porcentajes

\begin{tabular}{|c|c|c|c|c|c|c|}
\hline \multirow[t]{2}{*}{ Edad } & Asiste & No asiste & Total & Asiste & No asiste & Total \\
\hline & \multicolumn{3}{|c|}{ Valores absolutos } & \multicolumn{3}{|c|}{ Porcentajes } \\
\hline 14 & 1929630 & 143073 & 2072703 & 93.00 & 7.00 & 100 \\
\hline 15 & 1795246 & 250240 & 2045486 & 87.77 & 12.23 & 100 \\
\hline 16 & 1479695 & 421347 & 1901042 & 77.84 & 22.16 & 100 \\
\hline 17 & 1330317 & 481791 & 1812108 & 73.41 & 26.59 & 100 \\
\hline 18 & 1134584 & 619466 & 1754050 & 64.68 & 35.32 & 100 \\
\hline Total & 7669472 & 1915917 & 9585389 & 80.00 & 20.00 & 100 \\
\hline
\end{tabular}

Fuente: cálculos propios con datos de la ENOE 2005-2016, INEGI (2016a).

En una comparación internacional con los países de la Organización para la Cooperación y Desarrollo Económicos (OCDE) y del G20, en el 2015 se observa que hubo países como Irlanda y Japón, donde 95 y 94\%, respectivamente, de los jóvenes de entre 15 y 19 años estudiaban, mientras que en el promedio de los países de la oCDE, la asistencia escolar era de $84 \%$. México se ubica entre los países con los porcentajes más bajos de asistencia escolar en el rango de edad de 15 a 19 años, con una tasa de asistencia de 56\% (ocDE, 2016).

$\mathrm{Al}$ realizar el desglose por nivel de ingreso, las diferencias son aún más marcadas. En la gráfica 1 se presenta la asistencia escolar del grupo de 14 a 18 años por decil de ingreso en México y se observa que 57\% de los jóvenes en este rango de edad del decil I asisten a la escuela, comparado con $85.7 \%$ de los jóvenes del decil X. La tasa más baja de asistencia se presenta entre los jóvenes de 18 ańos del decil I, donde sólo asiste a la escuela 24.7\%. 
Gráfica 1. México. Tasa de asistencia escolar de los jóvenes de 14 a 18 años por decil de ingreso en 2014

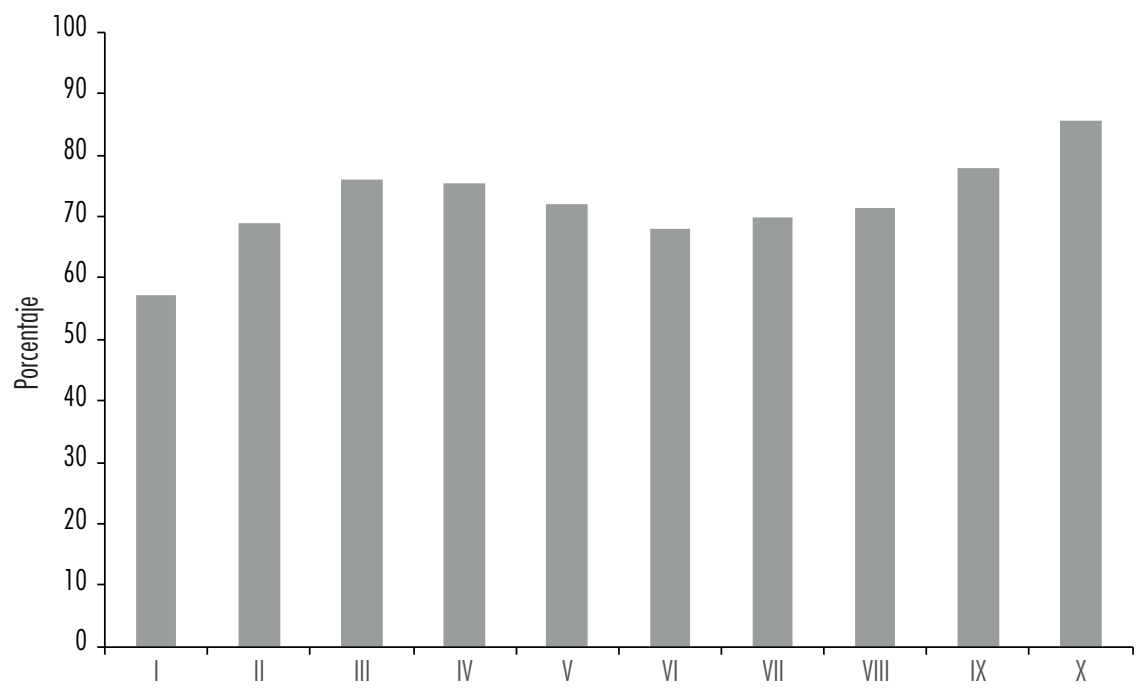

Fuente: cálculos propios con datos de la ENOE, INEGI (2015).

\section{REVISIÓN DE LITERATURA}

\section{Una breve aproximación teórica}

Según Sen (1992), la equidad implica la igualdad en cierto atributo, más no en todos. Entonces, para llegar a ser una sociedad equitativa, ¿qué variable o atributo se debe igualar? En forma muy resumida, se puede decir que hay tres enfoques principales que responden a esta interrogante, dependiendo si la igualdad se da en: $i$ ) el bienestar, si se considera que el objetivo es maximizar la suma total de utilidad de una sociedad; ii) los bienes o recursos; para Rawls (1971) debe existir igualdad en bienes primarios (como derechos, libertades y oportunidades; ingreso y riqueza), mientras para Dworkin (1981), la igualdad se debe dar en los recursos, es decir, que cada individuo esté satisfecho con su dotación (o cesta) de bienes; iii) las oportunidades, donde se consideran, entre otros, el enfoque de capacidades señaladas por Sen y el enfoque de "nivelar el piso" (o "alisar el camino") de Roemer (Formichella, 2011).

En el enfoque de capacidades de Sen, el criterio a igualar son las capacidades o libertades que tienen las personas para elegir su propio modo de vida; mientras que en el enfoque de Roemer, se requiere que haya igualdad en las 
oportunidades; aquí se considera que los resultados de un individuo son consecuencia de acciones que él lleva a cabo, y que están determinadas por dos tipos de circunstancias: las que el individuo puede controlar, y las que salen fuera de su control (Formichella, 2011). De esta forma, se hace una distinción entre la desigualdad ocasionada por la responsabilidad individual o factores sobre los que se tiene control, lo que Roemer (1998) llama "esfuerzo", y la desigualdad causada por circunstancias predeterminadas sobre las que el individuo no tiene control, como la raza, el género, las condiciones familiares, el capital cultural, las herencias, el ingreso familiar, entre otros. Así, la desigualdad de oportunidades se relaciona con las circunstancias que quedan fuera del control de la persona (Paes et al., 2008).

Dada la importancia en el desarrollo de un individuo, cierto nivel de educación es necesario para establecer la igualdad de oportunidades entre individuos, pero ¿cómo se mide este nivel o la igualdad de oportunidades en educación? Según López et al. (2017), en la literatura se identifican dos formas para medir la desigualdad educativa: la desigualdad en el acceso a la educación (e.g. los años de escolaridad) y el rendimiento académico (e.g. el desempeño del estudiante). Mientras algunos trabajos empíricos estudian una de estas vertientes, otros estudian ambas, pues según Gamboa y Waltenberg (2015) ambos aspectos son importantes y están interrelacionados, por lo que deberían medirse simultáneamente.

\section{Literatura de trabajos empíricos}

Algunos trabajos internacionales señalan que las circunstancias predeterminadas juegan un rol importante en la desigualdad de oportunidades educativas y económicas. Ferreira y Gignoux (2014) encontraron que la desigualdad de oportunidades por circunstancias predeterminadas explica hasta $35 \%$ de las diferencias en el logro educativo y que el género, la educación de la madre y la ocupación del padre son variables que muestran resultados robustos para explicar la desigualdad educativa. En el mismo sentido, Paes et al. (2008) encontraron que la educación de la madre y la ocupación del padre son causas importantes tanto de la desigualdad educativa como de la desigualdad en los ingresos de los adultos. Mientras que Gamboa y Waltenberg (2012) consideran que la educación de los padres y el tipo de escuela a la que asisten los jóvenes de 15 años en seis países de América Latina son factores importantes de desigualdad de oportunidades, no así el género. 
Otros trabajos internacionales abordan en concreto la desigualdad educativa condicionada por antecedentes familiares (circunstancias sobre las que un individuo no tiene control), y encuentran que los ingresos familiares son un determinante importante en las oportunidades de educación. Haveman y Wolfe (1995) indican que el nivel educativo que alcanza una persona está determinado por los ingresos de la familia y la inversión que hace en educación, así como que la deserción escolar de los jóvenes de entre 15 y 19 años está determinada principalmente por problemas económicos.

Papay et al. (2015) encuentran grandes brechas en el logro educativo entre estudiantes de bajo y de alto ingreso en Massachusetts, Estados Unidos, y que las brechas son más amplias en niveles educativos mayores, incluyendo las tasas de graduación de educación media superior (EMS) y la entrada a la universidad. Reardon (2011) y Bailey y Dynarski (2011) también encontraron brechas en el logro académico entre niños y jóvenes que crecen en familias de bajos ingresos y aquellos que se crían en familias con ingresos más altos en Estados Unidos. Acemoglu y Pischke (2001) consideran que el ingreso familiar tiene un gran efecto en la matrícula universitaria, específicamente que un aumento en el ingreso familiar de $10 \%$ está asociado a un incremento de 1.4 puntos porcentuales en la probabilidad de asistir cuatro ańos a la universidad.

Gasparini (2002) encuentra que una mayor disparidad en el ingreso del hogar se transforma en mayores disparidades en las elecciones escolares en Argentina; mientras que González et al. (2014) señalan que un ingreso bajo del hogar reduce la probabilidad de demandar educación media superior o superior en Colombia, y que los jóvenes que provienen de hogares con ingresos altos tienen una probabilidad positiva y creciente en el tiempo de permanencia en la escuela.

Por otra parte, Ibáñez et al. (2020) consideran que a mayor edad, ser económicamente activo y habitar en un hogar con bajo clima educativo, será menor la probabilidad de asistencia escolar de los adolescentes en Argentina; mientras que la probabilidad de asistir es mayor entre las mujeres, los que cuentan con cobertura de salud y cuyos padres trabajan en el mercado laboral formal.

En México se han realizado estudios que analizan el problema de la desigualdad educativa utilizando datos de los censos (Martínez Rizo, 2002; Solís, 2010; Navarro y Favila, 2013), o de la Encuesta Nacional de Ingreso Gasto de los Hogares (ENigh) (De la Luz y Díaz, 2010; Martínez et al., 2011; Rangel y Ramírez, 2011). Algunos de estos trabajos muestran una asociación entre las características socioeconómicas de los individuos y los años de escolaridad alcanzados (Martínez Rizzo, 2003; Solís 2010; Solís et al., 2013; Navarro y 
Favila, 2013), o entre el ingreso familiar y la matrícula escolar (De la Luz y Díaz, 2010). En específico, Solís (2010) encontró que en México, la mayor parte de la variación en los años de escolaridad es explicada por los orígenes de clase (el capital económico y cultural de la familia de origen). De la Luz y Díaz (2010) indican que el nivel de ingresos es un determinante en las decisiones de educación del grupo poblacional de 15 a 23 ańos y que un aumento de $10 \%$ en el ingreso de las familias, incrementa la probabilidad de estar matriculado en $2.6 \%$ (en 2000), $2.5 \%$ (en 2002) y 2.4\% (en 2004).

En el mismo sentido, utilizando la Encuesta de Transición a la Educación Media Superior para el Distrito Federal, Solís et al. (2013) indican que los jóvenes del cuartil inferior del índice de orígenes sociales (IOS) tienen una probabilidad de continuar estudiando la EMs de 0.55 , frente a una probabilidad de 0.85 que tienen los jóvenes del cuartil superior. Por otra parte, en la Encuesta Nacional de Deserción en la Educación Media Superior (ENDEMs) (SEP, 2012), se encontró que entre las principales causas para abandonar la EMS están los factores económicos (falta de dinero en el hogar para útiles, pasajes o inscripción), de forma que los ingresos del hogar sí parecen ser un determinante de que un joven siga estudiando o no en México. Por su parte, Vargas y Valadez (2016) utilizan datos de la Encuesta Nacional de Juventud 2010 y modelos de regresión de Cox, y encuentran que el riesgo de abandonar la escuela está asociado indirecta y significativamente con la calidad educativa y con el estatus económico del adolescente.

Pese a que la literatura sobre el efecto del ingreso familiar en la asistencia escolar en México es abundante, al parecer no se ha estudiado esta relación a través de un panel longitudinal de un año, información que es más robusta para lidiar con probables problemas de simultaneidad. Además de esta aportación, el presente estudio permite reforzar la idea de una brecha intrageneracional basada en el ingreso familiar, con información específica que puede contribuir a focalizar políticas públicas a favor de la educación y de la disminución de la desigualdad educativa.

\section{METODOLOGÍA, VARIABLES Y DATOS}

\section{Metodología de estimación: modelo de regresión probit}

Modelar la asistencia escolar estadísticamente presenta la particularidad de que la variable de respuesta (asistencia) no es continua, sino más bien discreta; es decir, se asiste o no se asiste. Por tal motivo no es conveniente utilizar los 
modelos de regresión lineal tradicionales porque éstos asumen continuidad en la variable dependiente.

Por lo anterior, se emplean métodos de regresión para variable dependiente binaria, en este caso se seleccionó el modelo probit. El enfoque consiste en modelar la probabilidad de que un joven de entre 14 y 18 ańos que asistía a la escuela en el periodo $t$ continúe asistiendo a la escuela un año después, es decir, en $t+1$, como función del ingreso del hogar en un periodo $t$ y otras variables de control a través de una distribución acumulada de probabilidad normal cuyo argumento es una combinación lineal de los determinantes de la variable de respuesta (Wooldridge, 2016).

En el contexto de un modelo probit se puede representar como:

$$
\operatorname{Pr}\left(E_{i t+1}=1 \text { dado } E_{i t}=1, X_{i t}\right)=\Phi\left(X_{i t}^{\prime} \beta\right)
$$

Donde Pr denota Probabilidad, $E_{i t}\left(E_{i t+1}\right)$ es un indicador binario que toma el valor de 1 si el joven $i$ asiste a la escuela en el periodo $t(t+1), \Phi$ denota la distribución acumulada de la normal y $X_{i t}$ es un vector de características propias del joven $i$ y su familia en el periodo $t$, tal que:

$$
X_{i t}^{\prime} \beta=\beta_{0}+\beta_{1} x_{1 t}+\beta_{2} x_{2 t}+\cdots .+\beta_{k} x_{k t}
$$

El cambio en la probabilidad de que el joven asista a la escuela cuando cambia una variable independiente $x_{j}$, lo que se llama respuesta marginal, es igual a:

$$
\Delta \operatorname{Pr}\left(E_{i t+1}=1 \text { dado } E_{i t}=1, X_{i t}\right)=\left[\phi\left(X_{i t}^{\prime} \beta\right) \beta_{j}\right] \Delta x_{j t}
$$

Donde $\phi$ es la densidad de la distribución normal. El coeficiente estimado $\beta_{j}$ y la respuesta marginal tienen el mismo signo, pero no son iguales. En este sentido, los coeficientes no tienen una interpretación directa, por lo que es importante evaluar el impacto de los regresores a través de las respuestas marginales y probar si son significativamente distintos de cero.

Dos comentarios adicionales del modelo. La variable dependiente y los regresores están desfasados, la asistencia escolar se refiere al periodo $t+1$, mientras los regresores están fechados en el periodo $t$. Esto es conveniente para romper cualquier posible simultaneidad de las variables, sobre todo con el ingreso familiar. Al usar el ingreso familiar rezagado, el del periodo $t$, éste ya está dado en $t+1$ de forma que no puede ser afectado por la asistencia escolar en $t+1$. 
Por último, a diferencia de los estudios de sección cruzada, pueden darse cambios en el tiempo en factores comunes a todos los individuos (e.g. crisis económica o desastre natural) que afecten la variable de respuesta. Por este motivo conviene incluir variables dicotómicas de los periodos de tiempo para capturar este tipo de efectos fijos.

\section{Variables}

A pesar de que el interés es estimar el efecto del ingreso familiar en la asistencia escolar, se incluyeron además otras variables que pueden ser importantes para explicar la asistencia escolar de los jóvenes, como las características del joven, de su familia, del entorno y de la temporalidad de las observaciones en el modelo. De esta forma se evitan sesgos por omisión de variables y se mejora la bondad del ajuste. A continuación, se describen las variables independientes del modelo.

Variables de características del joven: se codificaron variables dicotómicas que toman el valor de 1 si se cumple la característica aludida y 0 si no se cumple, estas variables son Hombre, Va tarde (cursa un año escolar menor al que debería), Estudia secundaria, Trabaja, Casado y Migrante. Se incluyó la Edad en años cumplidos.

Variables de la familia: se probaron las siguientes variables dicotómicas, Hogar ampliado (vive algún familiar adicional), Tiene hermanos mayores, Tiene hermanos menores, Tiene ambos padres, Madre trabaja. Se incluyó la Educación de la madre (años de estudio), el Número de hermanos y el Ingreso familiar per cápita, este último consiste en los ingresos de todos los miembros del hogar con excepción del joven, expresados en pesos del 2016 y en términos per cápita.

Variables del entorno y de tiempo: se incluyeron 31 variables dicotómicas por estado y una para zonas rurales, 10 dummies de año y 3 dummies de trimestre.

\section{Datos}

Este trabajo utiliza datos de la ENOE desde el primer trimestre de 2005 hasta el tercer trimestre de 2016. La ENOE tiene una estructura de panel rotativo en la que se realizan entrevistas a los mismos hogares e individuos por cinco trimestres consecutivos, renovando cada trimestre a un quinto del total de la muestra (INEGI, 2016b). 
Estudios internacionales emplean paneles de datos con el historial de un individuo a lo largo de su vida escolar y sus características personales y familiares para medir el efecto del ingreso familiar en la asistencia escolar de los hijos; sin embargo, en México no se cuenta con datos panel de ese tipo, por lo que la ENOE - Con su estructura de panel rotativo-, se convierte en el mejor instrumento disponible que nos provee de microdatos desglosados por edad, estado, sexo, condición rural-urbana, características individuales, características del hogar, etcétera, para un mismo individuo a lo largo de un año de su vida (cinco trimestres consecutivos). Se consideran sólo a jóvenes de entre 14 y 18 años que viven con sus padres al momento de la primera entrevista. De esta manera, se cuenta con una muestra de 252077 observaciones en total.

La base de datos se organizó de la siguiente manera: cada trimestre se selecciona a los individuos que tienen entre 14 y 18 años que viven con sus padres, que están estudiando y que participan por primera vez en la entrevista, y se registran sus características individuales y de su hogar; utilizando su identificador personal, se unen la información de la primera y quinta entrevista de cada joven seleccionado. De esta forma, en la misma observación se tienen las características del joven y de su hogar reportadas en la entrevista inicial (periodo $t$ ), como su edad, sexo, número de integrantes de su hogar o su ingreso familiar y la asistencia escolar del joven en la entrevista final (periodo $t+1)$. Cada trimestre incluye un grupo diferente de jóvenes con información de su entrevista inicial y con su condición de asistencia escolar registrada en su entrevista final.

En el cuadro 2 se muestra la dinámica de asistencia escolar de los jóvenes entre su primera y su última entrevista. Del total de la muestra, 69.7\% (175 798 jóvenes) reportaron asistir a la escuela al momento de la última entrevista. Del total de jóvenes que reportaron estar estudiando en su primera entrevista, $86.5 \%$ continuaron estudiando un ańo después, mientras que entre los que reportaron no estar estudiando en su primera entrevista, $13.7 \%$ regresaron a la escuela un año después.

Cuadro 2. Dinámica de asistencia escolar de los jóvenes de 14 a 18 años

\begin{tabular}{lrcr}
\hline & Asiste en entrevista final & No asiste en entrevista final & \multicolumn{1}{c}{ Total } \\
\hline Asiste en entrevista inicial & $167859(86.5 \%)$ & $26088(13.5 \%)$ & $193947(100 \%)$ \\
No asiste en entrevista inicial & $7939(13.7 \%)$ & $50191(86.3 \%)$ & $58130(100 \%)$ \\
Total & $175798(69.7 \%)$ & $76279(30.3 \%)$ & $252077(100 \%)$ \\
\hline
\end{tabular}

Fuente: cálculos propios con datos de la ENOE 2005-2016, INEGI (2016a). 
En el cuadro 3 se presentan las medias para la muestra total, para los individuos que seguían asistiendo a la escuela un año después y para los que no siguieron asistiendo. Cabe recordar que en el modelo se utilizan las características del joven y de su hogar al momento de la entrevista inicial. Dado que este estudio se acota a aquellos jóvenes que asistían a la escuela en la entrevista inicial, el tamaño de la muestra se reduce a 193947 jóvenes.

Cuadro 3. Tabla de medias de las variables independientes

\begin{tabular}{|c|c|c|c|c|c|c|}
\hline Observaciones & $\begin{array}{c}\text { Total } \\
193947\end{array}$ & $\begin{array}{c}\text { Asiste en } \\
\text { entrevista final } \\
167859\end{array}$ & $\begin{array}{l}\text { No asiste en } \\
\text { entrevista final } \\
26088\end{array}$ & & & \\
\hline & Media & Media & Media & Min. & Max. & $\begin{array}{l}\text { Diferencia } \\
\text { estadística }\end{array}$ \\
\hline Hombre & $51 \%$ & $50 \%$ & $53 \%$ & 0 & 1 & $\star \star \star \star ~$ \\
\hline Edad en años & 15.7 & 15.6 & 16 & 14 & 18 & $\star \star \star$ \\
\hline Va tarde & $15 \%$ & $13 \%$ & $26 \%$ & 0 & 1 & 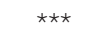 \\
\hline Secundaria & $40 \%$ & $40 \%$ & $42 \%$ & 0 & 1 & $\star \star \star ~$ \\
\hline Trabaja & $14 \%$ & $13 \%$ & $22 \%$ & 0 & 1 & $\star \star \star$ \\
\hline Casado & $3 \%$ & $0 \%$ & $1 \%$ & 0 & 1 & $\star \star \star$ \\
\hline Migrante & $11 \%$ & $11 \%$ & $10 \%$ & 0 & 1 & $\star \star \star ~$ \\
\hline Rural & $22 \%$ & $20 \%$ & $31 \%$ & 0 & 1 & 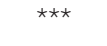 \\
\hline Hogar ampliado & $11 \%$ & $11 \%$ & $15 \%$ & 0 & 1 & $\star \star \star$ \\
\hline Tiene hermanos mayores & $54 \%$ & $53 \%$ & $56 \%$ & 0 & 1 & $\star \star \star$ \\
\hline Tiene hermanos menores & $65 \%$ & $65 \%$ & $69 \%$ & 0 & 1 & 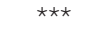 \\
\hline Número de hermanos & 3 & 3 & 3.3 & 1 & 14 & $\star \star \star ~$ \\
\hline Tiene ambos padres & $83 \%$ & $84 \%$ & $81 \%$ & 0 & 1 & $\star \star \star$ \\
\hline Educación de la madre & 8.3 & 8.6 & 6.4 & 0 & 26 & $\star \star \star \star ~$ \\
\hline La madre trabaja & $47 \%$ & $47 \%$ & $43 \%$ & 0 & 1 & 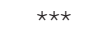 \\
\hline Ingreso familiar per cápita ${ }^{a}$ & 2.2 & 2.3 & 1.74 & 0.6 & 123.7 & $\star \star \star ~$ \\
\hline
\end{tabular}


Aunque todas las comparaciones simples de las medias de los que asistían y no asistían a la escuela son significativamente distintas de 0 al $1 \%$, las diferencias son más notables en unas cuantas variables. En particular aquellos que no asistían a la escuela en la entrevista final, en comparación a los que sí lo hicieron, tienen una incidencia mayor entre los que van rezagados en su carrera escolar, los que trabajan, los que residen en zonas rurales, los que tienen madres con menor educación y los que viven en hogares con ingresos reales per cápita menores. Las diferencias son menores, sin embargo apreciables, entre los hombres, los que cursan secundaria, los que viven en hogares ampliados y quienes no viven con ambos padres.

Al momento de la entrevista inicial, el ingreso familiar per cápita en los hogares de los jóvenes de la muestra era de MXN\$2 213 de 2016 al mes. En el grupo de jóvenes que reportaron no asistir a la escuela en la entrevista final, el ingreso familiar per cápita era en promedio de MXN\$1 745 al mes, es decir, MXN\$468 por persona menos. En el cuadro 4 se puede observar que el ingreso familiar per cápita de la media de la muestra se encuentra entre el decil VI y VII, mientras que el ingreso familiar per cápita de los jóvenes que dejaron de asistir a la escuela se encuentra entre el decil V y VI.

Cuadro 4. Ingreso familiar promedio per cápita mensual por decil y percentil de ingreso (México, 2016) (MXN\$)

\begin{tabular}{cccc}
\hline Decil & $\begin{array}{c}\text { Ingreso promedio } \\
\text { per cápita }\end{array}$ & Percentil & $\begin{array}{c}\text { Ingreso promedio } \\
\text { per cápita }\end{array}$ \\
\hline I & 290.50 & 10 & 559.91 \\
II & 722.16 & 20 & 880.28 \\
III & 1017.70 & 30 & 1149.25 \\
IV & 1300.43 & 40 & 1464.51 \\
V & 1623.78 & 50 & 1781.51 \\
VI & 1929.48 & 60 & 2102.88 \\
VII & 2348.00 & 70 & 2591.05 \\
VIII & 2864.97 & 80 & 3199.39 \\
IX & 3671.58 & 90 & 4205.77 \\
X & 7124.19 & 100 & 51763.45 \\
\hline
\end{tabular}

Fuente: cálculos propios con datos de la ENOE 2005-2016, INEGI (2016a). 
El cuadro 5 divide el porcentaje de jóvenes que reportaron asistir a la escuela en la entrevista final, dado que asistían a la escuela en la entrevista inicial, según diferentes características. Por ejemplo, de los hombres que asistieron a la escuela en la entrevista inicial, $85 \%$ reportó continuar asistiendo en la entrevista final, mientras que esto sucede para $87 \%$ de las mujeres.

Cuadro 5. Porcentaje de jóvenes de 14 a 18 años que asiste a la escuela en la entrevista final dado que asistían a la escuela en la entrevista inicial

\begin{tabular}{llll}
\hline Hombre & $85 \%$ & Mujer & $87 \%$ \\
16 años o menos & $89 \%$ & 17 años o más & $79 \%$ \\
Secundaria & $85 \%$ & Preparatoria & $86 \%$ \\
Trabaja & $79 \%$ & No trabaja & $87 \%$ \\
Casado & $62 \%$ & Soltero & $86 \%$ \\
Migrante & $88 \%$ & No migrante & $86 \%$ \\
Rural & $80 \%$ & Urbano & $88 \%$ \\
Hogar ampliado & $82 \%$ & Hogar nuclear & $86 \%$ \\
Tiene hermanos mayores & $85 \%$ & No tiene hermanos mayores & $86 \%$ \\
Tiene hermanos menores & $85 \%$ & No tiene hermanos menores & $87 \%$ \\
Dos hermanos o menos & $88 \%$ & Tres hermanos o más & $84 \%$ \\
Tiene ambos padres & $86 \%$ & No tiene padre o madre & $84 \%$ \\
Madre con secundaria o menos & $83 \%$ & Madre con preparatoria o más & $94 \%$ \\
La madre trabaja & $87 \%$ & La madre no trabaja & $85 \%$ \\
\hline
\end{tabular}

Fuente: cálculos propios con datos de la ENOE 2005-2016, INEGI (2016a).

La retención, es decir, la proporción de jóvenes que asistían a la escuela en la entrevista final, dado que lo hacían en la inicial, tiende a ser mayor entre los menores a 16 años en comparación a los de 17 o 18 años (esta diferencia es consistente con un resultado muy común en la literatura donde la deserción escolar se incrementa con la edad); los que no trabajan; los solteros; los que viven en un hogar nuclear (comparado con uno ampliado); y con madres con educación de preparatoria o más.

Se observa además que el porcentaje de jóvenes que asistían a la escuela en la primera entrevista aumenta a través de los años, al pasar de $72 \%$ en 2005 a $80 \%$ en 2015; mientras que el porcentaje de jóvenes que asistía a la escuela en la segunda entrevista, dado que asistía en la primera entrevista, 
también aumentó, aunque en menor proporción, al pasar de 84 a $87 \%$ en el mismo periodo. Por su parte, un desglose de asistencia por estado, indica que Coahuila y Michoacán presentan el menor porcentaje de asistencia en la primera entrevista de jóvenes de entre 14 y 18 años, con 64\% de asistencia en promedio; mientras que la Ciudad de México reporta la mayor tasa de asistencia con $85 \%$. En cuanto al porcentaje de retención, es decir, jóvenes que asistían a la escuela en ambas entrevistas, Baja California Norte y Ciudad de México reportan las tasas más altas, 90 y $89 \%$, respectivamente; mientras que Guanajuato tiene la tasa más baja con $80 \%$.

\section{RESULTADOS}

El cuadro 6 muestra los efectos marginales de la regresión probit, donde se consideran siete características particulares del joven, siete características de su hogar, incluido el ingreso familiar per cápita, y dos características particulares de la madre del joven. ${ }^{1}$ Como variables de control se incluyeron en la regresión 31 dummies de entidad federativa, 10 dummies de ańo y 3 dummies de trimestre; los efectos marginales de las variables de control no se presentan en el cuadro.

En general se obtuvo una $\mathrm{R}^{2}$ de McFadden de 0.0872 y una Count $\mathrm{R}^{2}$ de 0.8610 . Todas las variables analizadas son significativas al menos al $95 \%$ de confianza con excepción de las variables si el joven tiene hermanos menores (90\% de confianza), si es migrante y si la madre trabaja (que no son significativas).

Siguiendo los resultados que se muestran en el cuadro 6, se observa que los varones tienen en promedio una probabilidad 1.10 punto porcentual menor que las mujeres de continuar asistiendo a la escuela un año después. Por su parte, cada año adicional de edad reduce la probabilidad del joven de continuar asistiendo a la escuela en 5.5 puntos porcentuales y estar cursando la secundaria en la entrevista inicial, la reduce en 8.3 puntos porcentuales. Estar en un ańo escolar menor al que le corresponde por edad, trabajar o estar casado, también reducen la probabilidad de los jóvenes de continuar asistiendo a la escuela un año después. De las características del hogar, la condición rural, el vivir en un hogar ampliado (con otros familiares además de padres y hermanos), y el número de hermanos, reducen la probabilidad de continuar asistiendo a la

1 Debido a que en $17 \%$ de los casos no se cuenta con información del padre, y para no perder estas observaciones, no se incluyen variables que indiquen la escolaridad o la condición laboral del padre, sólo se considera la información concerniente a la madre. 
escuela un año después; mientras que tener hermanos mayores o menores y vivir con ambos padres, incrementan dicha probabilidad. La escolaridad de la madre también incrementa la probabilidad del joven de continuar asistiendo a la escuela, mientras que la condición laboral de la madre no tiene un efecto en dicha probabilidad. ${ }^{2}$

Por su parte la variable relevante en este trabajo, el ingreso familiar, tiene un efecto positivo de 1 punto porcentual. Es decir, un incremento de mil pesos al mes en el ingreso familiar per cápita, evaluado en la media (MXN\$2 213 per cápita), aumenta la probabilidad del joven de continuar asistiendo a la escuela un año después, en 1 punto porcentual. Evaluado en la media este efecto parece pequeño; sin embargo, al calcular el efecto marginal para los percentiles de ingreso 10,20,30, hasta 90, es posible observar importantes efectos positivos del ingreso en la probabilidad de continuar estudiando entre los jóvenes económicamente menos favorecidos.

La probabilidad de que un joven de 14 a 18 años continúe estudiando dado que se encuentra en el percentil 10 de ingreso es de $92 \%$, mientras que la probabilidad de continuar estudiando para un joven del percentil 20 aumenta a 97\%, para los jóvenes del percentil 30 es casi $99 \%$ y para el resto, es muy cercana al 100\% (véase gráfica 2). Esto es, entre más pobre es la familia de un joven, el efecto del ingreso en la probabilidad de que el joven continúe estudiando es mayor. De tal forma que, si el ingreso promedio per cápita de la familia de un joven del percentil 10 pudiera elevarse al ingreso promedio per cápita de una familia del percentil 20, la probabilidad del joven de continuar en la escuela se elevaría en 5 puntos porcentuales. Y si el ingreso promedio per cápita de este joven del percentil 10 aumentara para ubicarse en el ingreso promedio per cápita del percentil 30, la probabilidad de dicho joven de continuar estudiando se elevaría en 7 puntos porcentuales.

El ingreso per cápita promedio de una familia en el percentil 10 es MXN\$559.91, mientras que en el percentil 20 es MXN\$880.28 y en el percentil 30 es MXN\$1 149.25 (véase cuadro 4). Entonces, aumentar la probabilidad de continuar estudiando de los jóvenes de 14 a 18 años del percentil 10 en 5 puntos porcentuales requeriría un aumento de $\mathrm{MXN} \$ 320.37$ per cápita en su ingreso familiar mensual; mientras que aumentar la probabilidad en 2 puntos porcentuales más requeriría un aumento adicional de MXN\$268.97 per cápita.

2 Para hacer más ligera la redacción, no se comenta en la interpretación de cada efecto marginal que, para evitar el problema de doble causalidad entre el ingreso y la asistencia escolar, las variables independientes están medidas en el periodo inicial $(t)$ mientras que la variable dependiente está medida un año después, en el periodo final $(t+1)$. 
Cuadro 6. Efectos marginales del modelo probit. Efecto de características individuales y del hogar en la probabilidad de que un joven de 14 a 18 años que vive en su hogar y que asistía a la escuela en t, continúe asistiendo a la escuela en ++1

\begin{tabular}{|c|c|c|}
\hline Hombre (d) & -0.0110 & 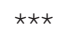 \\
\hline Edad en $†$ & -0.0549 & $\star \star \star$ \\
\hline Va tarde en $\dagger(\mathrm{d})$ & -0.0217 & $\star \star \star$ \\
\hline Secundaria 0 menos en $\uparrow(d)$ & -0.0826 & $\star \star \star$ \\
\hline Trabaja en $†$ (d) & -0.0362 & $\star \star \star$ \\
\hline Casado en $\uparrow(d)$ & -0.1218 & $\star \star \star$ \\
\hline Migrante en $†$ (d) & 0.0078 & \\
\hline Rural en $\dagger(d)$ & -0.0344 & $\star \star \star$ \\
\hline Hogar ampliado en $\dagger(d)$ & -0.0140 & $\star \star \star *$ \\
\hline Tiene hermanos mayores (d) & 0.0078 & $\star \star$ \\
\hline Tiene hermanos menores (d) & 0.0061 & * \\
\hline Número de hermanos & -0.0113 & $\star \star \star$ \\
\hline Vive con ambos padres en $\dagger$ (d) & 0.0105 & $\star \star \star$ \\
\hline Años de educ. de la madre & 0.0093 & $\star \star \star \star ~$ \\
\hline Trabaja la madre en $†$ (d) & -0.0032 & \\
\hline Ingreso familiar pc en $\dagger$ & 0.0102 & $\star \star \star$ \\
\hline$N$ & 155623 & \\
\hline Wald Chi ${ }^{2}$ & 4839.17 & \\
\hline McFadden's R2 & 0.0872 & \\
\hline Count $R^{2}$ & 0.8610 & \\
\hline
\end{tabular}

Notas: efectos Marginales. (d) para variables dummy. ${ }^{\star} \mathrm{p}<0.10$; ${ }^{\star \star} \mathrm{p}<0.05$; ${ }^{\star * \star} p<0.01$; Ingreso en pesos de 2016. La muestra se acota a los jóvenes de 14 a 18 años que viven con su familia, que asistían a la escuela en la primera entrevista ( $†$ ) y que reportan el ingreso familiar.

Fuente: cálculos propios con datos de la ENOE 2005 a 2016, INEGI (2016a). 
Gráfica 2. Probabilidad de que un joven de 14 a 18 años que asistía a la escuela en t, continúe asistiendo en t+1 según percentil de ingreso familiar

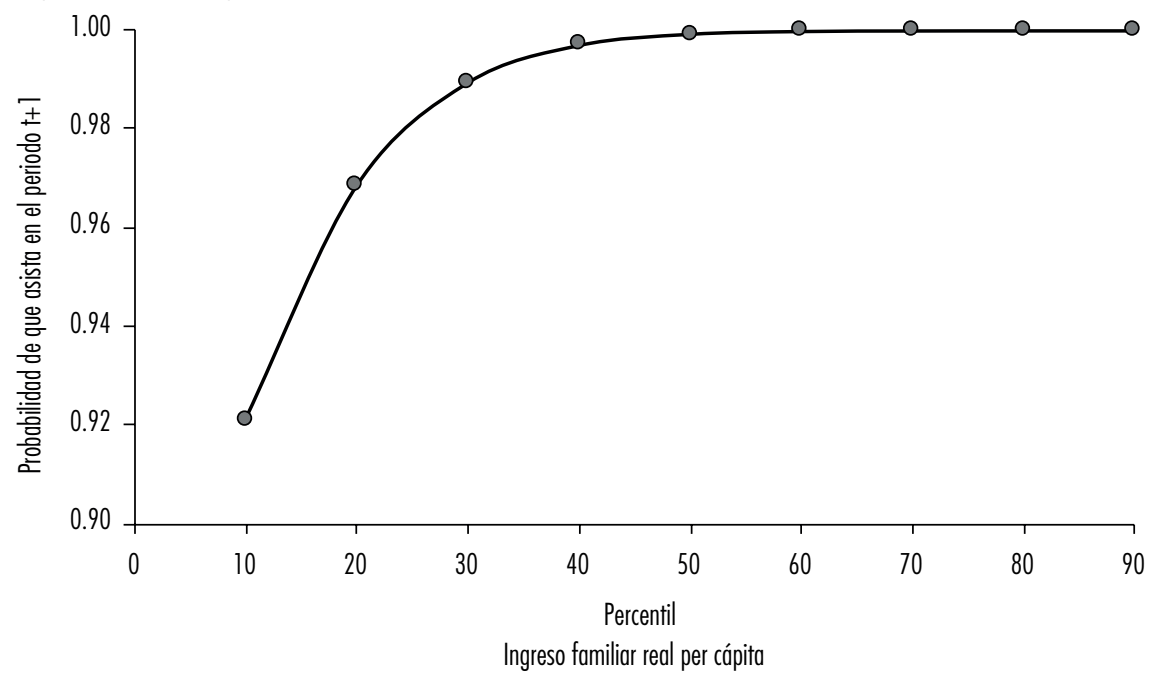

Fuente: cálculos propios con datos de la ENOE 2005-2016, INEGI (2016a).

La gráfica 3 nos muestra una interacción del sexo con el ingreso per cápita por percentiles. Como puede observarse, la probabilidad de continuar estudiando para los hombres de 14 a 18 años es menor que para las mujeres, principalmente en los primeros 5 percentiles de ingreso. Esta brecha se va cerrando conforme el ingreso per cápita familiar es mayor. Así, mientras en los percentiles 10 y 20 la brecha entre mujeres y hombres es de 2 puntos porcentuales en el percentil 40 es de menos de 1 punto porcentual.

En la gráfica 4 se presenta una interacción del ingreso con la edad por percentiles; obsérvese que en los percentiles de ingreso más bajo hay una amplia brecha en la probabilidad de continuar en la escuela que crece con la edad. La probabilidad de continuar estudiando de un joven de 14 ańos del percentil 10 es $98 \%$, mientras que para un joven de 18 ańos del mismo percentil es de $81 \%$, es decir, 17 puntos porcentuales de diferencia. El efecto de la edad prácticamente desaparece a partir del percentil 60 de ingreso.

Aún sin considerar los percentiles de ingreso, el efecto de la edad es muy importante, pues mientras la probabilidad de continuar estudiando de un joven de 14 años es $93 \%$, para un joven de 18 años es de $70.5 \%$, es decir, una diferencia de 22.5 puntos porcentuales. 
Gráfica 3. Probabilidad de que un joven de 14 a 18 años que asistía a la escuela en t, continúe asistiendo en t+1 por sexo, según percentil de ingreso familiar

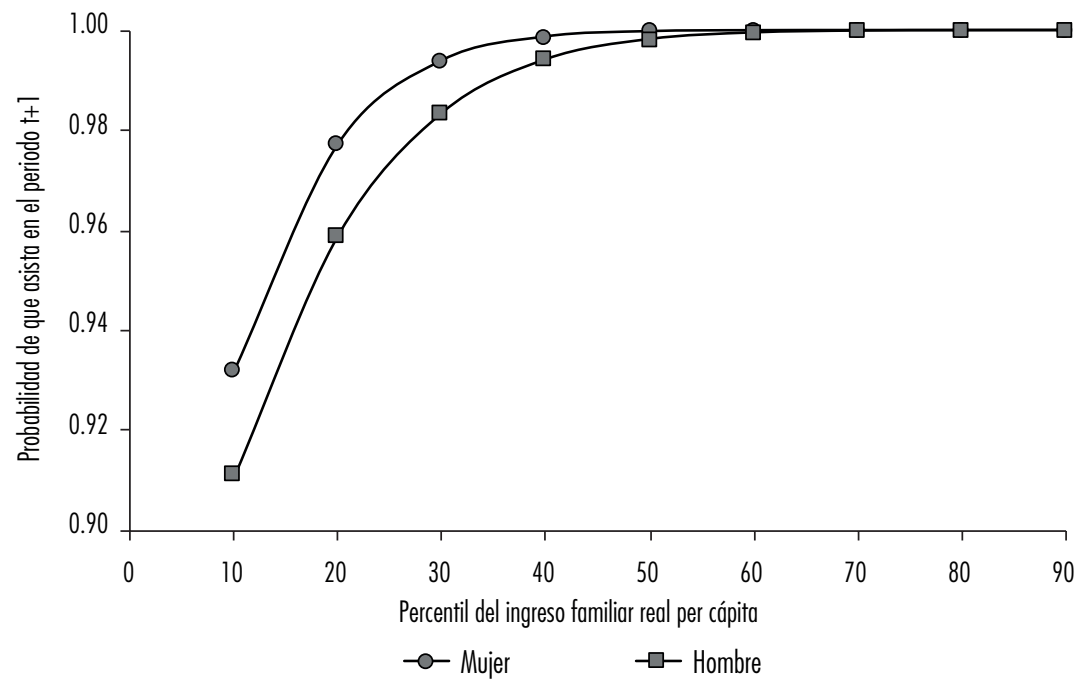

Fuente: cálculos propios con datos de la ENOE 2005-2016, INEGI (2016a).

Gráfica 4. Probabilidad de que un joven de 14 a 18 años que asistía a la escuela en t, continúe asistiendo en t+1 por edad, según percentil de ingreso familiar

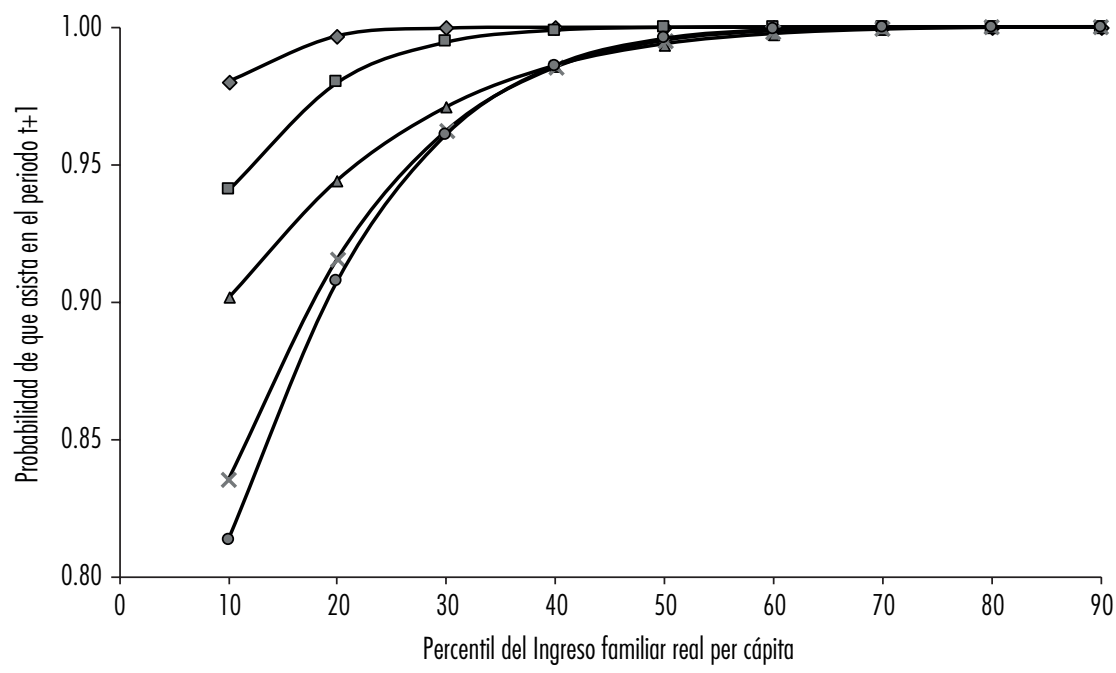

$\checkmark$ Edad $=14 \quad \rightarrow-$ Edad $=15 \quad \rightarrow-$ Edad $=16 \quad *$ Edad $=17 \quad \rightarrow-$ Edad $=18$

Fuente: cálculos propios con datos de la ENOE 2005-2016, INEGI (2016a). 
Finalmente, en la gráfica 5 se presenta una interacción del ingreso con la condición rural-urbana por percentiles. Como puede observarse, la probabilidad de continuar estudiando para un joven de 14 a 18 ańos que vive en el área rural es menor que para un joven que vive en la ciudad, y esta brecha se cierra hasta el percentil 90. De nueva cuenta, la brecha es más amplia en los primeros deciles de ingreso, un joven de 14 a 18 años del percentil 10 o 20 que vive en el área rural, tiene 6 puntos porcentuales menos de probabilidad de continuar estudiando que un joven del mismo percentil de ingresos que vive en el área urbana. Si no se consideran las diferencias en la distribución del ingreso, en promedio un joven de entre 14 y 18 años del área rural, tiene 4 puntos porcentuales menos de probabilidad de continuar estudiando que un joven del área urbana.

Gráfica 5. Probabilidad de que un joven de 14 a 18 años que asistía a la escuela en t, continúe asistiendo en t+1 por condición rural-urbana, según percentil de ingreso familiar

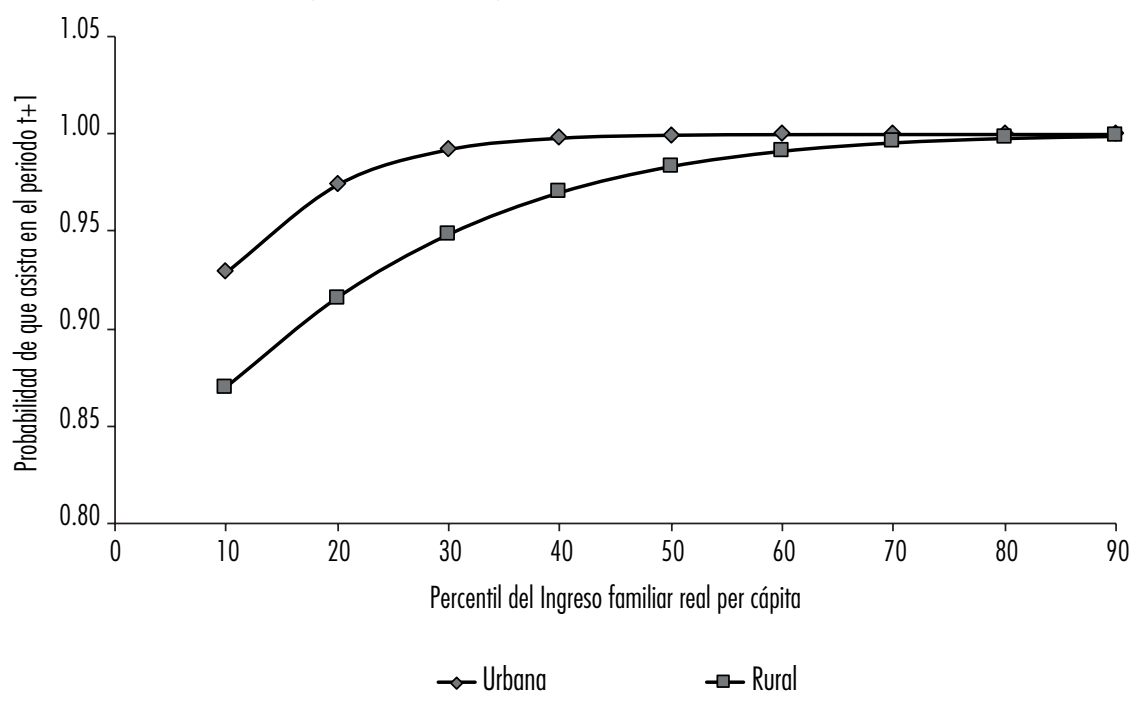

Fuente: cálculos propios con datos de la ENOE 2005-2016, INEGI (2016a).

\section{CONCLUSIONES}

Utilizando la ENOE de 2005 a 2016, este trabajo estudia si el ingreso familiar per cápita tiene un impacto en la probabilidad de que los jóvenes de 14 a 18 ańos que viven con sus padres y asisten a la escuela, continúen haciéndolo 
un año después. Para corregir el sesgo en las estimaciones originado por la doble causalidad entre el ingreso familiar y la asistencia escolar de los jóvenes, se aprovecha la estructura de panel rotativo de la ENOE y se utilizan las características del joven y de su hogar, incluyendo el ingreso familiar, con un año de rezago.

Los resultados obtenidos indican que el ingreso familiar predice la probabilidad de que un joven continúe asistiendo a la escuela en México. En concreto, después de controlar por otras características del joven y de su familia, la probabilidad de que un joven continúe estudiando es mayor entre mayor sea el ingreso familiar. Además, este efecto positivo del ingreso familiar en la probabilidad de continuar estudiando es relativamente importante en los primeros deciles de ingreso. Es decir, una transferencia de dinero incrementa más la probabilidad de continuar asistiendo a la escuela si se otorga a los jóvenes de familias más desfavorecidas en comparación a los de hogares más prósperos.

La presencia de un efecto positivo del ingreso familiar sobre la asistencia escolar de los jóvenes, refuerza la idea de la desigualdad de oportunidades educativas y la existencia de una brecha intrageneracional en educación basada en el ingreso familiar en México. Los resultados de este estudio nos muestran que, los jóvenes de 14 a 18 años de los deciles de ingreso más bajos, y sobre todo si son hombres o viven en el área rural, son quienes tienen menores probabilidades de continuar asistiendo a la escuela. En el mismo sentido, la brecha en la probabilidad de asistencia escolar entre hombres y mujeres y entre jóvenes rurales y urbanos son más amplias en los deciles más bajos de ingreso, cerrándose conforme el ingreso familiar per cápita aumenta.

Dado que la desigualdad de oportunidades se relaciona con las circunstancias que quedan fuera del control de la persona (Paes et al., 2008), y en la medida que la desigualdad se deba a factores que los individuos no controlan, como la desigual dotación transmitida por la familia, es justo que el poder público intente reducir, tanto como sea posible, tal desigualdad de condiciones (Piketty, 2014). En este sentido, la desigualdad de oportunidades justifica la intervención pública a través del diseño de políticas públicas encaminadas a "nivelar el campo de juego" (Roemer, 1998) por medio de acciones y recursos que permitan que todos tengan las mismas condiciones, en particular en el grupo de jóvenes de los deciles más bajos de ingresos, los jóvenes que viven en el área rural, los jóvenes del sexo masculino y los jóvenes de mayor edad. 


\section{BIBLIOGRAFÍA}

Acemoglu, D. y Pischke, J.S. (2001), "Changes in the wage structure, family income, and children's education", European Economic Review, vol. 45, núm. 4, DOI <https://doi.org/10.1016/S0014-2921(01)00115-5>

Bailey, M.J. y Dynarski, S.M. (2011), "Inequality in post-secondary education", en G. J. Duncan y R.J. Murnane (eds.), Whither opportunity? Rising inequality, schools, and children's life chances, New York, Russell Sage Foundation.

Beck, U. (1998), La sociedad del riesgo. En camino hacia otra sociedad moderna, Madrid, Paidós.

Comisión Económica para América Latina y el Caribe (CEPAL) (2008), Juventud y cohesión social en Iberoamérica: un modelo para armar, Chile, CEPAL. Recuperado de <https:/www.cepal.org/es/publicaciones/3639-juventudcohesion-social-iberoamerica-un-modelo-armar>

De Gregorio, J. y Lee, J.W. (2002), "Education and income inequality: new evidence from cross-country data", Review of Income and Wealth, vol. 48, núm. 3, DoI <https://doi.org/10.1111/1475-4991.00060>

De la Luz Tovar, C. y Díaz González, E. (2010), "Dispersión del ingreso y demanda de educación media superior y superior en México", Análisis Económico, vol. XXV, núm. 58. Recuperado de <http://www.redalyc.org/ articulo.oa?id=4131308300>

Dworkin, R. (1981), "What is equality? Part 2: equality of resources", Philosophy and Public Affairs, vol. 10, núm.4, New Jersey, Princeton University Press, Autumn.

Ferreira, F.H.G. y Gignoux, J. (2014), "The measurement of educational inequality: achievement and opportunity", The World Bank Economic Review, vol. 28, núm. 2, Washington, D.C., Oxford University Press, May.

Formichella, M. M. (2011), "Análisis del concepto de equidad educativa a la luz del enfoque de las capacidades de Amartya Sen”, Educación, vol. 35, núm. 1, Costa Rica, Universidad de Costa Rica. Recuperado de <https:// www.redalyc.org/pdf/440/44018789001.pdf>

Gamboa, L. y Waltemberg, F. (2012), "Inequality of opportunity for educational achievement in Latin America: Evidence from PISA 2006-2009", Economics of Education Review, vol. 31, núm. 5, DOI <https://doi.org/10.1016/j. econedurev.2012.05.002>

y Waltemberg, F. (2015), "Measuring inequality of opportunity in education by combining information on coverage and achievement in 
PISA", Educational Assessment, vol. 20, núm. 4, DOI <https://www.tandfonline.com/doi/full/10.1080/10627197.2015.1093926>

Gasparini, L.C. (2002), "On the measurement of unfairness: an application to high school attendance in Argentina", Social Choice and Welfare, vol. 19, núm. 4, DOI <https://doi.org/10.1007/s003550200156>

González, C.G., Mora, J.J. y Cuadros, A.F. (2014), "Oportunidades educativas y características familiares en Colombia: un análisis por cohortes", Revista de Economía del Rosario, vol. 17, núm. 1, DOI <http://dx.doi. org/10.12804/rev.econ.rosario.17.01.2014.06>

Gruber, J. (2009), "Introduction: What have we learned about the problems of and prospects for disadvantaged youth?”, en J. Gruber (ed.), The problems of disadvantaged youth: an economic perspective, Chicago, University of Chicago Press.

Haveman, R. y Wolfe, B. (1995), “The determinants of children's attainments: a review of methods and findings", Journal of Economic Literature, vol. 33, núm. 4, United States, American Economic Association, December.

Hopenhayn, M. (2008), "Inclusión y exclusión social en la juventud latinoamericana”, Pensamiento Iberoamericano, núm. 3, Madrid, Agencia Española de Cooperación Internacional y Fundación Carolina. Recuperado de $<$ https://dialnet.unirioja.es/servlet/articulo?codigo=2781553>

Ibáñez Martín, M., Formichella, M. y Costabel, L. (2020), "Exclusión social: explorando la dimensión educativa en Argentina", Problemas del Desarrollo. Revista Latinoamericana de Economía, vol. 51, núm. 200, DOI <https:// doi.org/10.22201/iiec.20078951e.2020.200.68518>

Instituto Mexicano de la Juventud (IMJ) (2007), Encuesta Nacional de Juventud 2005, México, Instituto Mexicano de la Juventud.

Instituto Nacional de Estadística y Geografía (INEGI) (2015), Microdatos de la Encuesta Nacional de Ocupación y Empleo, 2005-2015. Recuperado de <http://www.beta.inegi.org.mx/proyectos/enchogares/regulares/enoe/ default.html>

Instituto Nacional de Estadística y Geografía (INEGI) (2016a), Microdatos de la Encuesta Nacional de Ocupación y Empleo, 2005-2016. Recuperado de $<$ http://www.beta.inegi.org.mx/proyectos/enchogares/regulares/enoe/default.html>

(2016b), Metodología de la Encuesta Nacional de Ocupación y Empleo. Recuperado de <http://www.beta.inegi.org.mx/proyectos/enchogares/regulares/enoe/default.html>

López, A., Virgüez. A., Silva, C. y Sarmiento, J. (2017), "Desigualdad de oportunidades en el sistema de educación pública en Bogotá Colombia", 
Lecturas de Economía, núm. 87, DOI <http://dx.doi.org/10.17533/udea. le.n87a06>

Mancha Torres, G. L. (2011), La desatención generacional: Los jóvenes frente a la escasez de oportunidades. Una propuesta de medición a través de indicadores. Nuevo León 2005-2009 (Tesis de Maestría), México, Universidad Autónoma de Nuevo León.

Martínez Jasso, I., Gómez Meza, M. V. y De la Garza Flores, R. (2011), "Educación y desigualdad del ingreso en México", en E. Aguayo Téllez y E. Rangel González (coord.), Capital humano, pobreza y distribución del ingreso en México, México, Plaza y Valdés/unnt.

Martínez Rizo, F. (2002), "Nueva visita al país de la desigualdad. La distribución de la escolaridad en México (1970-2000)", Revista Mexicana de Investigación Educativa, vol. 7, núm. 16, septiembre, México, Consejo Mexicano de Investigación Educativa, A.C. Recuperado de <http://www. redalyc.org/articulo.oa?id=14001603>

(2003), Calidad y equidad en educación. 20 años de reflexiones, México, Santillana.

Miranda, A. y Salvia, A. (1998), "Exclusión de los jóvenes en la década de los 90. Factores, alcances y perspectivas", Papeles de Población, vol. 4, núm. 16, abril-junio, México, Universidad Autónoma del Estado de México. Recuperado de <http://www.redalyc.org/pdf/112/11201612.pdf>

Navarro Chávez, J.C. y Favila Tello, A. (2013), "La desigualdad de la educación en México, 1990-2010: el caso de las entidades federativas", Revista Electrónica de Investigación Educativa, vol. 15, núm. 2, mayo, México, Instituto de Investigación y Desarrollo Educativo de la Universidad Autónoma de Baja California. Recuperado de <http://redie.uabc.mx/vol15no2/ contenido-navarro-favila.html>

Organización Internacional del Trabajo (огт) (2005), El empleo de los jóvenes: vias para acceder a un trabajo decente, Informe VI, Ginebra, oIT. Recuperado de <http://www.ilo.org/youthmakingithappen/PDF/rep-vi_es.pdf>

Organización para la Cooperación y el Desarrollo Económicos (OCDE) (2016), Education at a Glance 2016: OECD Indicators, París, OECD Publishing. Recuperado de <https://www.oecd-ilibrary.org/education/education-at-aglance-2016_eag-2016-en>

Paes de Barros, R., Ferreira, F. H. G., Molinas Vega, J.R. y Saavedra Chanduvi, J. (2008), Midiendo la desigualdad de oportunidades en América Latina y el Caribe, Washington, Banco Mundial. Recuperado de <http://siteresources.worldbank.org/LACINSPANISHEXT/Resources/Book_IOH.pdf> 
Papay, J. E., Murnane, R.J. y Willett, J.B. (2015), “Income-based inequality in educational outcomes: learning from state longitudinal data systems", Educational Evaluation and Policy Analysis, vol. 37, núm. 1, DOI < https:// doi.org/10.3102/0162373715576364>

Piketty, T. (2014), El capital en el siglo XXI, México, Fondo de Cultura Económica.

Rangel González, E. y Ramírez Grimaldo, N.C. (2011), "Desigualdad educativa en México”, en E. Aguayo Téllez y E. Rangel González (coord.), Capital humano, pobreza y distribución del ingreso en México, México, Plaza y Valdés/UANL.

Rawls, J. (1971), Teoría de la Justicia, México, FCE.

Reardon, S.F. (2011), "The widening academic achievement gap between the rich and the poor: new evidence and possible explanations", en G. J. Duncan and R.J. Murnane (eds.), Whither opportunity? Rising inequality, schools, and children's life chances, New York, Russell Sage Foundation.

Roemer, J. E. (1998), "Igualdad de oportunidades", Isegoría. Revista de filosofía moral y politica, núm. 18, DOI < https://doi.org/10.3989/isegoria.1998. i18>

Secretaría de Educación Pública (sEP) (2012), Reporte de la Encuesta Nacional de Deserción en la Educación Media Superior (ENDEMS) 2011, México, SEP. Recuperado de <http://www.sems.gob.mx/work/models/sems/Resource/10787/1/images/Anexo_6Reporte_de_la_ENDEMS.pdf>

Sen, A. (1992), Inequality reexamined, Cambridge, Cambridge University Press.

Solís, P. (2010), "La desigualdad de oportunidades y las brechas de escolaridad", en A. Arnaut y S. Giorguli (coords.), Educación. Los grandes problemas de México, vol. 7, México, El Colegio de México.

Solís, P., Rodríguez Rocha, E. y Brunet, N. (2013), "Orígenes sociales, instituciones, y decisiones educativas en la transición a la educación media superior. El caso del Distrito Federal", Revista Mexicana de Investigación Educativa, vol. 18, núm. 59, octubre-diciembre, México, Consejo Mexicano de Investigación Educativa, A.C. Recuperado de <http://www.redalyc. org/articulo.oa?id=14028945005>

Tilak, J. (2002), "Education and poverty", Journal of Human Development, vol. 3, núm. 2, DOI <https://doi.org/10.1080/14649880220147301>

Tilly, C. (2003), "Changing forms of inequality", Sociological Theory, vol. 21, núm. 1, DOI <https://doi.org/10.1111/1467-9558.00173> 
Gloria Mancha y Edgardo Ayala

Vargas, E. D. y Valadez, A. (2016), "Calidad de la escuela, estatus económico y deserción escolar de los adolescentes mexicanos", Revista Electrónica de Investigación Educativa, vol. 18, núm. 1, enero, México, Instituto de Investigación y Desarrollo Educativo de la Universidad Autónoma de Baja California. Recuperado de <http://redie.uabc.mx/redie/article/view/713> Wooldridge, J. M. (2016), Introductory econometrics: A modern approach, $6^{\text {th }}$ Ed. Boston, Cengage Learning. 